\title{
Vertical Transportation: Effects of Harmonics of Drives by PM Machines
}

Research Article

\author{
R. Anand ${ }^{1}$, B. Gayathridevi ${ }^{1}$, B.K. Keshavan² \\ ${ }^{1}$ Department of Electrical Engineering, PES Institute of Technology, Bangalore, India, \\ ${ }^{2}$ Department of Electrical Engineering, PES University, Bangalore, India
}

Received September 07, 2018; Accepted October 06, 2018

Abstract: Permanent magnet motor drive is a widely used technology, offering many advantages, such as exceptional speed, torque control and greater flexibility. Improvement of reliability and efficiency has become a great research interest. Towards this direction and taking into account the major developments in permanent machine technology over the recent years, the use of energy recovery converters has been introduced in various industrial applications. In this paper, the effects of harmonics on a three-phase motor controlled by a drive are analysed, and the behaviours of the filter topology after adopting regenerative drives are studied. The main contribution of this study is a methodology to foresee the standards that can be achieved with the use of an active frontend system topology with filters. Moreover, the use of an optimum filter that eases the power system distortion is presented. The analysis presented in this paper is validated experimentally.

Keywords: Elevators $\bullet$ Permanent magnet synchronous machine $\bullet$ Adjustable speed drive $\bullet$ Quality $\bullet$ Safety $\bullet$ Reliability $\bullet$ Robustness

\section{Introduction}

The world that we live in and survive in now would almost certainly have been immobile had it not embraced innovations in mass transportation. The basic Ward Leonard drive system for direct current (DC) motors was invented and introduced in 1893, and 100 years later, the variable-voltage and variable-frequency drive (VVFD) technology evolved for alternating current (AC) motors, which came into existence for vertical transportations. During the past 30 years, the adoptability of AC VVFDs has grown (Holtz and Qi, 2013), and for many years, they have become the inherent and preferred way of controlling vertical transportation traction machines. The advantages, which include increased system efficiency, simplified testing and commissioning, as well as improved passenger ride quality, are well documented by many researchers. Increasingly, the issues of power quality, voltage variations, injection of harmonics (Burch R F, 2006; and IEEE Standard 519, 1981) onto the equipment and the electric system are becoming points of concern when selecting and sizing elevator systems.

\section{Vertical transportation}

The focus on elevators has become more prominent and critical; the areas that need analysis are adjustable speed motor drive and its performance analysis with reference to the latest trend of brushless DC motors or permanent magnet synchronous motor (PMSM) (Anand and Mahesh, 2016a, 2016b, 2016c) and Chattered Institution of Building Services Engineers (CIBSE, 2010). In the present scenario, where the world is heading towards skyscrapers and is going extra-vertical, the elevator has become the most basic, common, essential and mandatory requirement that contributes to about $6 \%-10 \%$ of the total energy consumption of a building, as analysed by Karlis (2014) and Nobile et al. (2014). 
It should also be noted that there are numerous adjustable speed drives (ASDs) adopted for enabling preciseness in controlling the speed profile schemes (which has effects on inter-harmonic theory), accuracy in deceleration, improvement in the time taken for the travel when it responds to a call from start to stop (which indeed generates numerous harmonics that are fed back to the supply source). The measure of harmonics is also being studied with the existing systems with prototype system integration; this would help the people in the society for optimisation and effective utilisation of energy, especially focussed more on the urban areas in our country. Fig. 1 represents the general taxonomy of the elevators, which are generally classified based on the requirements by the buyers; the requirements are driven mostly based on the occupancy and movement of men and materials in the buildings.

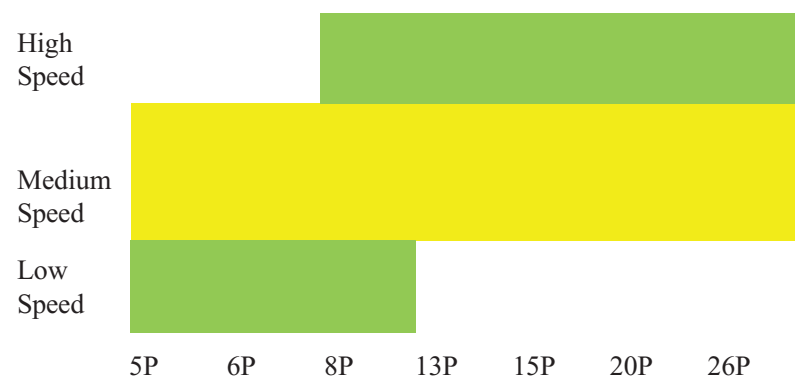

Fig. 1. Classification of elevators according to market segments (low speeds are $\leq 1 \mathrm{~m} / \mathrm{s}$; medium speeds are $>1 \mathrm{~m} / \mathrm{s}$ and $<2.5 \mathrm{~m} / \mathrm{s}$; high speeds are $\geq 2.5 \mathrm{~m} / \mathrm{s}$ )

Modern equipment with PMSM drive has the ability to control velocity and other critical parameters of the motion system, as explained by Kulkarni et al. (2000), such as rate of acceleration, harmonics, wide range of output frequencies and rate of jerk. Additionally, techniques in cases of indirect vector control have the capacity for efficiency optimisation during dynamic loadings, discussed by Anand and Mahesh (2016a, 2016b, 2016c) and Loannis Karatzaferis et al. (2017).

Elevators that are equipped and driven directly by PMSMs and with harmonic orders during operation of a specific equipment, such as a non-controlled six-pulse rectifier, have more demand in medium-rise buildings, as described by Alfio Consoli et al. (2012) and Jinping He et al. (2011). Generally, buildings that are less than $30 \mathrm{~m}$ in height are equipped with a direct coupling drive that is gear oil free. A medium-rise building's elevator is adopted and designed to carry a load of 300-700 kg at a speed of $0.20-1 \mathrm{~m} / \mathrm{s} ; 3-7.5 \mathrm{~kW}$ would be the rating of the elevator driving machines with speed of the shaft $<100 \mathrm{rpm}$. Higher-capacity elevator machines and high-speed elevator drives would require a higher rating of the driving capability, which can be rated as high as $500 \mathrm{~kW}$ (Anand and Mahesh, 2016a, 2016b, 2016c). Jerks experienced for a PMSM-driven elevator are significant enough to suppress transient vibration, as analysed by Yukinori Inoue et al. (2012) and Vukosavic et al. (2003).

The reasons behind the rapid growth in VVFDs are controllability, good dynamic performance and flexibility. Since the use of VFDs has become such a popular solution for industry, their improvement has become a research interest. There are numerous electric drive-controlled applications, such as tooling machines, packaging machines, etc., which are characterised by frequent stops/starts per time unit and intermittent duty cycles. Some of the main considerations are reliability and efficiency, as well as their improvement. The world that we survive in now would almost certainly have been impractical had it not embraced innovations in transportation. Typical VFDs usually implement unidirectional mains rectification and dissipate the regenerated kinetic energy via braking resistors, discussed by Huayong Yang et al. (2007). This can result in significant energy loss, especially when start/stops are frequent. Another important issue is the ride-through capability: in critical processes, the drive should withstand voltage sags that may last for periods ranging from a grid cycle up to several minutes. This can be critical in specific industrial processes in which a single interruption can severely affect the production. Several solutions have been proposed, usually using bidirectional converters that connect an appropriate energy storage technology to the DC bus of the drive, so that the load's kinetic or potential energy can be recovered and used again when necessary. An analytical investigation on the optimal torque that should be applied during transient speed for better energy recovery is presented. However, the loss model for both the electrical and the mechanical losses is quite simple, and moreover, only a limited number of load cases is covered. 
The six-pulse VVFD is most commonly applied in industrial applications, because it has been proven to be costeffective and reliable. A six-pulse VVFD, shown in Fig. 2, consists of three main sections: the input diode bridge, intermediate DC bus capacitors and output transistors. The input stage is made up of a three-phase full-wave diode bridge that rectifies the incoming three-phase AC power into DC power. Since the diodes allow current to pass only in one direction, it should be noted that the input stage of a six-pulse VVFD acts as a one-way street - allowing electrical energy to flow into the drive but not back into the line. The intermediate stage, or 'DC bus', is primarily made up of capacitors. The capacitors filter the voltage ripple created by the AC/DC conversion and act as a buffer by storing energy.

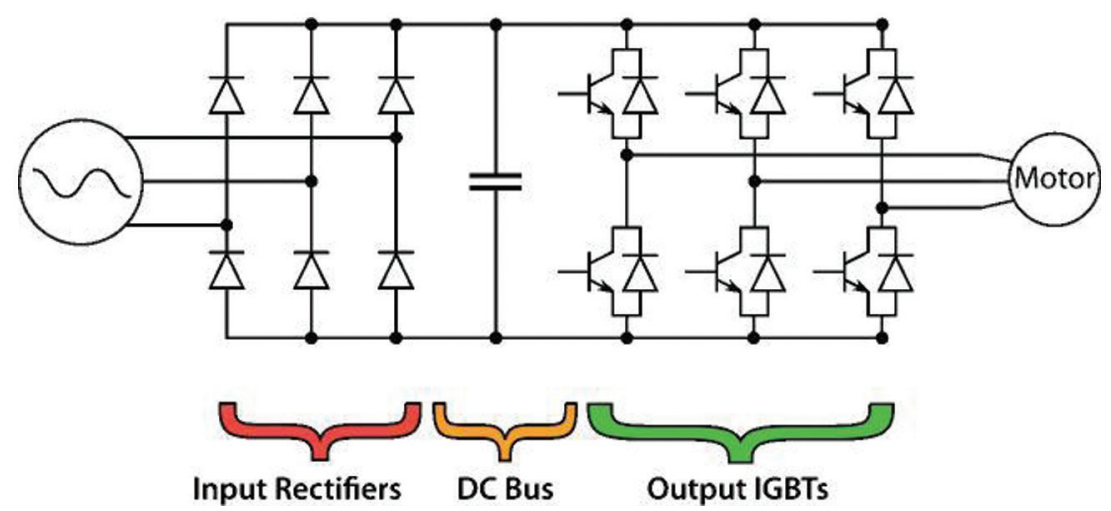

Fig. 2. Six-pulse WFD

The output stage of the VVFD consists of six insulated-gate bipolar transistors (IGBTs), which turn on and off at a high frequency $(\geq 8 \mathrm{kHz}$ ), thereby modulating the $\mathrm{DC}$ voltage from the bus capacitors. The technology is referred to as pulse-width modulation (PWM) and allows the drive to construct a three-phase variable AC voltage/frequency output to the elevator motor. By controlling the magnitude and phase of the voltage and frequency, the drive is able to control the motor's torque and speed. Unlike the input diodes, current can flow in both directions across the IGBTs, allowing energy to be put back onto the DC bus capacitors. An electrical load in which the power is consumed or delivered is generally classified by the nature of the current draws, i.e. linear and non-linear loads. Each harmonic current component is at a particular frequency and the sum of the fundamental frequency and the harmonic is the current required by the load, as shown in Equation 1:

$$
I_{r m s}=I_{\text {fundamental }}+\sum_{n=2}^{\infty} I_{n}
$$

\section{Drives and their effects}

According to the Institute of Electrical and Electronics Engineers (IEEE), harmonics are defined as the sinusoidal components of a periodic wave or quantity having frequencies that are integral multiples of the fundamental frequency. The harmonics of a $60 \mathrm{~Hz}$ or $50 \mathrm{~Hz}$ utility power supply would include the frequencies of the fundamental, first, third, fifth, seventh and so on continued up to the fortieth.

The IEEE 519-1992 standard, titled 'IEEE Recommended Practices and Requirements for Harmonic Control in Electrical Power Systems', developed from a need to define and measure acceptable system-level voltage and current harmonics. The utility company is responsible for providing its customers with clean sinusoidal voltage with low distortion. In turn, the customers have a responsibility to not create too many current harmonics and, thus, cause undesirable voltage distortion for other customers. The IEEE 519 standard provides a basis for measuring harmonics and defines acceptable harmonic levels. Any non-linear load's impedance does change throughout the AC electrical cycle, and the load draws current irregularly. Since the irregular current draw is not sinusoidal, it 
contains harmonics of the fundamental frequency. The current harmonics act on the system impedances within a building's electric power system. As the number and magnitude of the harmonics increases, the voltage available to other loads within the system becomes distorted. Eventually, if the distortion is high enough, the operation of other equipment can be adversely affected. In short, higher current harmonics result in a greater root mean square (RMS) current draw from the electric system. The increased current means the sourcing equipment (fuses, wires and transformers) of the system must be oversized. Additionally, as the harmonic currents flow through resistive impedances, additional heat is lost.

Specified in the IEEE 519 standard, the point where the utility's electric system and the consumer's system come together is defined as the point of common coupling (PCC). Literally, it is the first point at which the electric system is distributed within the consumer's facility, typically the facility's main feeder panel. The PCC is where all the loads within the consumer's system come together to present a unified load to the utility. This is important because some of the consumer's loads may actually compensate or negate the lower power factor or high harmonics of other loads. As a result, the utility sees an aggregate of all consumer loads at the PCC. The distortion of the collective load measured at the PCC is often better than the distortion of any one individual non-linear load.

That being said, the degree to which a load affects the utility depends largely on the relative size and duty of that load. A single 100-hp, VVFD-driven chiller running at continuous duty will have a much greater impact on the building's electric system and utility load than a $40-\mathrm{hp}$ elevator system operating at $50 \%$ duty, at best. The harmonic currents from the chiller VVFD alone could exceed the total current drawn from the elevator VVFD, making the elevator load somewhat inconsequential. However, since the facility's main feeder panel is often hard to access by equipment contractors, a general practice has been adopted to apply the terms and conditions of the IEEE 519 standard to the local accessible electrical system.

This is typically the disconnect switch feeding the equipment in question. So, in practice, the PCC becomes the disconnect switch for the chiller system. Likewise, the disconnect switch for the elevator system is the PCC for the elevator control supplier and contractor. While this is not technically correct according to the standard (and, further, the utility would never make such a measurement), it is the accepted practice. The theory is that if the power quality at the equipment disconnect is in compliance, then the equipment can in no way negatively affect the power quality within the building. Consider that an elevator is fed from an individual isolation transformer that is on the line side of the PCC. The transformer is sized based on the volt-amperes (VA) of the elevator motor (where transformer $\mathrm{VA}=$ full-load amperage [FLA] of motor $\times$ volts of motor $\times 1.732$ ). If the transformer has impedance around $5 \%$, the resulting ratio of the short circuit current to the demand current (ISC/IL) will be around 20. Acceptable total demand distortion (TDD) values would be $5 \%$ if the ratio is $<20$ and $8 \%$ if $\geq 20$. Note that this would be considered a minimum sizing for the transformer, and the configuration would not typically be used. Normally, the input FLA to the controller would be considered when sizing the transformer, which would push the VA of the transformer higher. The result is that the ISC/IL ratio would increase to approximately $25-30$, and the acceptable TDD value would be $8 \%$.

During a typical elevator cycle, energy is put into the system, stored and returned. During the motoring mode, energy flows from the utility to the motor, and mechanical work is done when the elevator cab moves. Additionally, both potential and kinetic forms of energy are stored in the elevator system and returned to the drive during the generating mode. Since the current cannot flow across the input rectifiers, the regenerated energy is stored in the DC bus capacitors. Traditionally, non-regenerative VVFDs and braking resistors have been used to rid the capacitors of the excess regenerated energy. As current flows into the capacitors, the voltage across the capacitors increases. When the DC bus voltage reaches a threshold, a resistor circuit is closed across the capacitors, and current flows through the braking resistor, dissipating the excess electrical energy as heat. The heat represents the energy loss in the system, since it cannot be recovered. Braking resistors are twice as inefficient when additional energy is needed to power an air-conditioning unit to cool the control room.

A regenerative unit can be used in lieu of a braking resistor, and the regenerated power can be returned to the grid, where it is consumed by other building loads. Regenerative units are becoming increasingly popular, as they can dramatically increase system efficiency and lower the total cost of elevator ownership. The components of regenerative drives are similar to those of a six-pulse VVFD, except there is no input diode stage. The regenerative drive has DC bus capacitors and an IGBT stage, used to channel the output current into the mainline. Additionally, an inductor is used to smooth current flow and synchronise the current to the voltage and frequency of the mainline. When the voltage of the coupled DC bus reaches a threshold, the regenerative unit's IGBT cycle at the same frequency of the line and current flows back onto the line. It should be noted that since the regenerative unit's IGBT's only cycle is at $60 \mathrm{~Hz}$, there are significantly less switching losses during operation than for the PWM operation of a 
VVFD. Since the regenerative IGBTs switch the current into the line in a similar manner as the diodes in a six-pulse rectifier, a regenerative unit will have similar harmonic content during the regenerative mode as a VVFD.

Elevator loads do change throughout the AC electrical cycle, and the load draws current irregularly depending on the cycle and duty. Since the irregular current draw is through the drives, which amounts to non-sinusoidal application, it contains harmonics of the fundamental frequency.

A series of low-pass harmonic filters can be placed at the input of a VVFD or regenerative unit to reduce the harmonic currents. The harmonic filter is composed of inductors and capacitors and designed such that the filter passes current at the fundamental frequency but blocks current at higher frequencies.

The active front end (AFE) system is composed of two back-to-back inverters. The first inverter synchronises itself to the line voltage and actively rectifies the AC voltage into DC. This active rectifier stage uses an inductive capacitive filter to link to the AC line; because the AFE uses PWM with a high carrier frequency, the switching losses are higher compared to the block-style commutation of the regenerative unit. The PWM iron losses in the AFE filter are also higher compared to those in similar-sized harmonic filters. AFE systems can only be connected to a balanced three-phase wye and a centre-grounded electric system; delta-connected systems are not permissible. Additionally, the phase voltages should be $\pm 5 \%$ with respect to each other. A transformer must be used in buildings having an older delta electric system or if the phases are greatly unbalanced.

The AFE performs very well in terms of power factor (PF) displacement and distortion, given by Equation (2):

$$
p f_{\text {true }}=\frac{P_{a v g}}{V_{r m s} I_{r m s}} \cdot \frac{1}{\sqrt{1+\left(\frac{T H i D}{100}\right)^{2}}}
$$

Table 1. Harmonics filters

\begin{tabular}{lccc}
\hline Drive topology & PF & THiD & Initial cost \\
\hline \hline AC WFD & 0.95 & $60 \%-80 \%$ & $\$-$ Low \\
AC WVFD with choke & 0.96 & $45 \%$ & $\$+$ - Above moderate \\
Regenerative with AC WFD and choke & 0.96 & $45 \%$ & $\$ \$-$ Moderate \\
AC WFD and isolation transformer & 0.96 & $45 \%$ & $\$ \$$ - Medium \\
Harmonic filter with AC WFD and regenerative unit & $0.99-1.00$ & $8 \%$ & $\$ \$$ - Medium \\
AC WFD with active front end & 1.00 & $3 \%-5 \%$ & $\$ \$$ - High \\
\hline
\end{tabular}

The result is current flowing from the line in a nearly pure sine wave with low harmonic distortion; in many cases, it can be $<2 \%$ of total harmonic current distortion (THiD). The PF, as shown in Equation 2, which consists of PF displacement and PF distortion, is regulated to a value close to unity, as shown in Table 1. Additionally, a high-quality electromagnetic interference filter (EMI) filter in front of the system is necessary, as the active rectifier creates a high level of common-mode EMI noise relative to earth ground. The second inverter functions as a motor drive and controls the motor as before with the passive filter and an improved filter system, wherein the inductance (Li) and capacitance $(\mathrm{C})$ are tuned close to the fifth harmonic dominant value and the other inductance (LC) connected avoided the fifth harmonics from other sources, as shown in the block diagram in Fig. 3. The series of Li, Lc and $\mathrm{C}$ sets the tuning frequency well below the fifth harmonics of the power system; the independence of the front-end stage is also captured in the system for tolerance with power quality analyser in Fig. 4. This shows the improvement, but at the additional cost for the EMI compliance controller with additional filters in the motion control sub-systems.

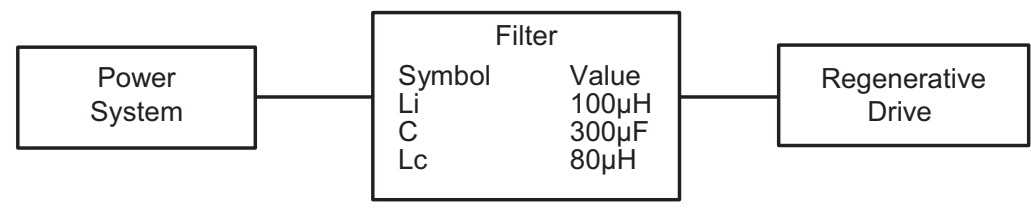

Fig. 3. Block diagram for filter 
An AFE exceeds the requirements of the IEEE 519 standard, but the added performance comes at a higher cost.
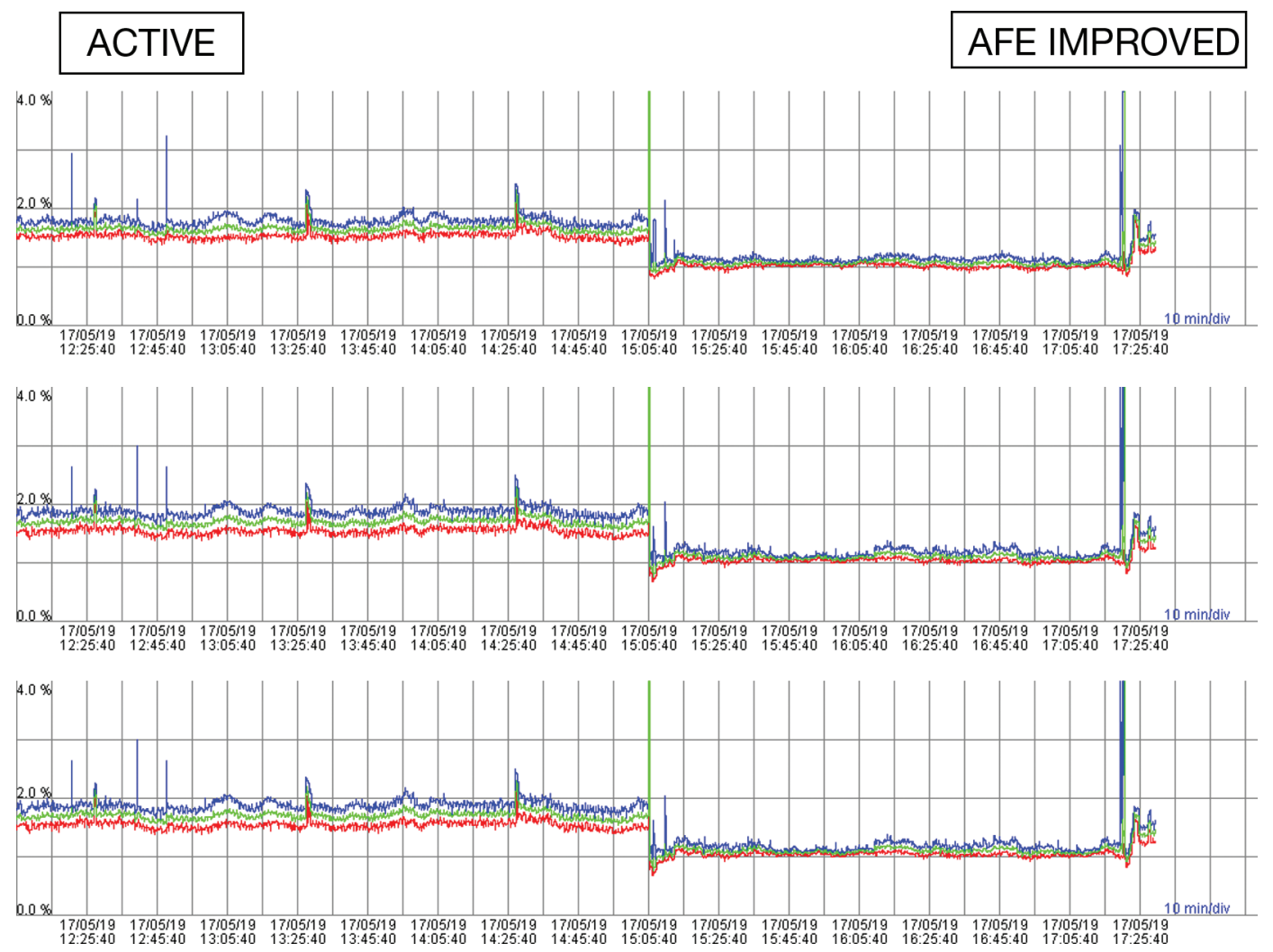

Fig. 4. Power quality captured in real time

\section{Conclusion}

Harmonic mitigation was carried out with a prototype in a real-time application using filters in the controllers. The extra components required for mitigating drive harmonics require more packaging and were integrated into the elevator controller, and the results were in line with the IEEE 519 standards.

\section{Inference and recommendations}

The IEEE 519 standard is meant as a guideline and provides goals for acceptable power quality. It is not economical or feasible that every elevator meet these requirements, as other loads within the building will have a dominating effect. In the end, a building owner or consultant will need to weigh the different drive technologies and paybacks to see whether the added investment of regenerative drives and harmonic mitigation justify the benefits. However, payback can ultimately be achieved in the form of smaller electrical components, regenerative energy, utility rebates and increased operating lifetime of other system components. 


\section{References}

Anand, R. and Mahesh, M. (2016a). Analysis of elevator drives energy consumptions with permanent magnet machines. In: Proceedings of the 2016 IEEE Smart Energy Grid Engineering (SEGE), Oshawa, ON, 21-24 August, pp. 186-190.

Anand, R. and Mahesh, M. (2016b). Drives analysis with dynamic loads on elevators and interactive study on integration systems. In: Proceedings of the 2016 IEEE International Conference on Power Electronics, Drives and Energy Systems (PEDES), Trivandrum, India, 14-17 December, pp. 1-6.

Anand, R. and Mahesh, M. (2016c). Elevator drives energy analysis with duty loads and behavior in dynamic conditions. In: Proceedings of the IEEE Emerging Technologies and Innovative Business Practices for the Transformation of Societies (EmergiTech), 3-6 August, pp. 133-138.

CIBSE (Chattered Institution of Building Services Department). (2010). Guide D. Transportation Structure in Buildings. London, UK: CIBSE.

Consoli, A., Scelba, G., Scarcella, G. and Cacciato, M. (2012). An Effective Energy-Saving Scalar Control for Industrial IPMSM Drives. IEEE Transactions on Industrial Electronics, 60(9), pp. 3658-3669.

He, J., Mao, C., Lu, J. and Yang, J. (2011). Design and Implementation of an Energy Feedback Digital Device Used in Elevator. IEEE Transactions on Industrial Electronics, 58(10), pp. 4636-4642.

Holtz, J. and Qi, X. (2013). Optimal Control of MediumVoltage Drives - An Overview. IEEE Transactions on Industrial Electronics, 60(12), pp. 5472-5481.

IEEE Recommended Practices and Requirements for Harmonic Control in Electrical Power Systems, IEEE Standard 519.

Inoue, Y., Morimoto, S. and Sanada, M. (2012). Control Method Suitable for Direct-Torque-Control Based
Motor Drive System Satisfying Voltage and Current Limitations. IEEE Transactions on Industry Applications, 48(3), pp. 970-976.

Karatzaferis, L., Tatakis, E., Papanikolaou, N. (2017). Investigation of Energy Savings on Industrial Motor Drives Using Bidirectional Converters. IEEE Access, 5, pp. 17952-17961.

Karlis, A. D. (2014). Energy consumption estimation on lift systems: the advantages of VVVF drives. In: Proceedings of the 2014 International Conference on Electrical Machines (ICEM), Berlin, Germany, September 2-5, pp. 751-755.

Kulkarni, A. B., Nguyen, H. and Gaudet, E. W. (2000). A comparative evaluation of fine regenerative and non-regenerative vector controlled drives for $A C$ gearless elevators. In: Conference Record of the 2000 IEEE Industry Applications Annual Meeting, Rome, Italy October 8-12, vol. 3, pp. 1431-1437

Burch, R. F.(2006). IEEE 519 Update From a Utility Perspective In: Conference Record of the 2005/2006 IEEE/PES Transmission and Distribution Conference and Exhibition, Dallas, USA, May 21-24, pp. 1162-1163

Nobile, G., Sciacca, A. G., Cacciato, M., Cavallaro, C., Raciti, A., Scarcellag, G. and Scelba, G. (2014). Energy harvesting in roped elevators. In: Proceedings of the 2014 International Symposium on Power Electronics, Electrical Drives, Automation and Motion, Ischia, Italy, 18-20 June, pp. 533-540.

Vukosavic, S. N. and Levi, E. (2003). Robust DSPBased Efficiency Optimization of Variable Speed Induction Motor Drive. IEEE Transactions on Industrial Electronics, 50(3), pp. 560-570.

Yang, H., Sun, W. and Xu, B. (2007). New Investigation in Energy Regeneration of Hydraulic Elevators. IEEE/ASME Transactions on Mechatronics, 12(5), pp. 519-526. 